EDITORA

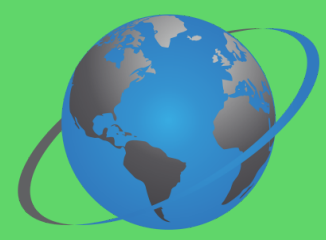

OMNIS SCIENTIA

\title{
SAÚDE PÚBLICA EM
}

\section{TEMPOS PANDEMICOS}

\section{VOLUME 1}

Organizador:

Raul Sousa Andreza

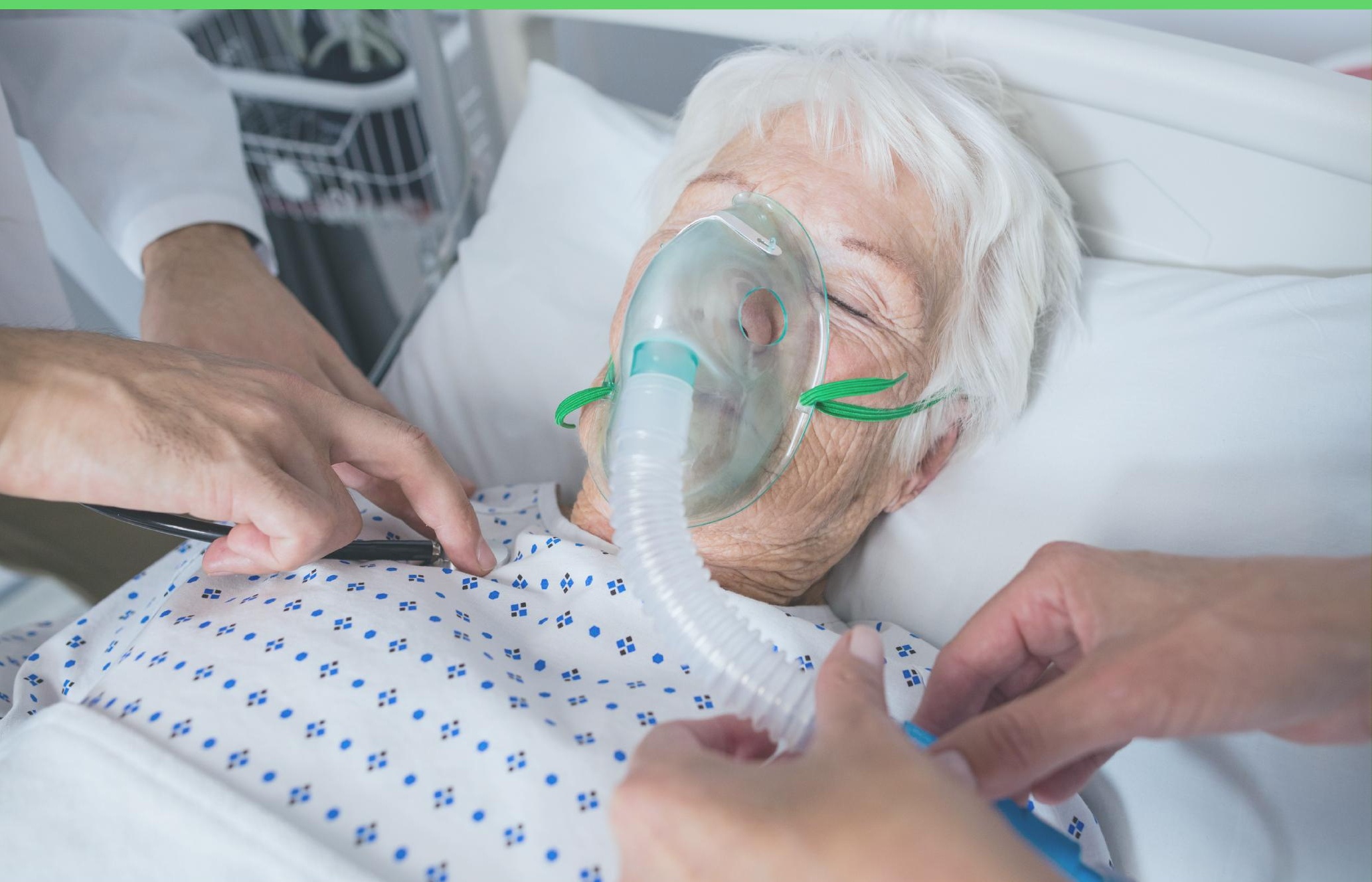


EDITORA

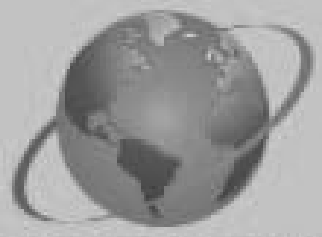

OMNIS SCIENTIA

\section{SAÚDEE PÚBUICA BM}

\section{THEMIPOS PANDENMICOS}

\section{VOLUME 1}

Organizador:

Raul Sousa Andreza

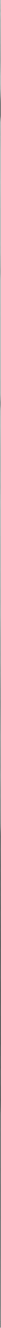


Editora Omnis Scientia

SAÚDE PÚBLICA EM TEMPOS PANDÊMICOS

Volume 1

$1^{a}$ Edição

TRIUNFO - PE

2021 


\section{Editor-Chefe}

Me. Daniel Luís Viana Cruz

Organizador (a)

Prof. Me. Raul Sousa Andreza

\section{Conselho Editorial}

Dra. Pauliana Valéria Machado Galvão

Dr. Wendel José Teles Pontes

Dr. Walter Santos Evangelista Júnior

Dr. Cássio Brancaleone

Dr. Plínio Pereira Gomes Júnior

Editores de Área - Ciências da Saúde

Dra. Camyla Rocha de Carvalho Guedine

Dra. Cristieli Sérgio de Menezes Oliveira

Dr. Leandro dos Santos

Dr. Hugo Barbosa do Nascimento

Dr. Marcio Luiz Lima Taga

Dra. Pauliana Valéria Machado Galvão

\section{Assistentes Editoriais}

Thialla Larangeira Amorim

Andrea Telino Gomes

Imagem de Capa

Freepik

\section{Edição de Arte}

Vileide Vitória Laranjeira Amorim

\section{Revisão}

Os autores

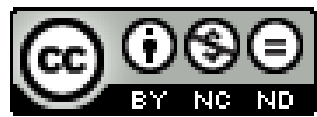

Este trabalho está licenciado com uma Licença Creative Commons - AtribuiçãoNãoComercial-SemDerivações 4.0 Internacional.

O conteúdo abordado nos artigos, seus dados em sua forma, correção e confiabilidade são de responsabilidade exclusiva dos autores. 
Dados Internacionais de Catalogação na Publicação (CIP) (eDOC BRASIL, Belo Horizonte/MG)

S255 Saúde pública em tempos pandêmicos [livro eletrônico] / Organizador Raul Sousa Andreza. - Triunfo, PE: Omnis Scientia, 2021.

286 p. : il.

Formato: PDF

Requisitos de sistema: Adobe Acrobat Reader

Modo de acesso: World Wide Web

Inclui bibliografia

ISBN 978-65-88958-41-4

DOI 10.47094/978-65-88958-41-4

1. Ciências da saúde. 2. Saúde pública. 3. Pandemia. I. Andreza, Raul Sousa.

CDD 610

Elaborado por Maurício Amormino Júnior - CRB6/2422

\author{
Editora Omnis Scientia \\ Triunfo - Pernambuco - Brasil \\ Telefone: +55 (87) 99656-3565 \\ editoraomnisscientia.com.br \\ contato@editoraomnisscientia.com.br
}




\section{PREFÁCIO}

A incidência do novo coronavírus no Brasil é preocupante. No entanto, a saúde pública do país e o sistema de atendimento visa abranger a diversidade que o Brasil apresenta, o sistema único de saúde (SUS) tem como base a integralidade, a universalidade e a equidade de todos os pacientes e trabalhadores. Instituído assim, para democratizar toda a saúde brasileira, tem o interesse de ofertar serviços de qualidade a população. Portanto, ao longo da história de sua consolidação a saúde pública foi deixado de lado e passou a gerar grandes problemas aos profissionais atuantes.

De fato, os estudos desenvolvidos no âmbito da saúde pública se propõem a articular conhecimentos de diferentes saberes e fazeres fornecendo subsídios teóricos, práticos e metodológicos que contribuem positivamente para a construção de estratégias e políticas públicas que visam o desenvolvimento de informações e ações em prol de uma saúde de qualidade para toda comunidade.

O presente livro é composto por 24 capítulos elaborados por autores pertencentes às ciências da saúde e suas áreas afins com o objetivo de proporcionar conhecimentos e compartilhar experiências e resultados de estudos desenvolvidos em várias localidades brasileiras e que visam à elucidação de diferentes situações de saúde.

Em nossos livros selecionamos um dos capítulos para premiação como forma de incentivo para os autores, e entre os excelentes trabalhos selecionados para compor este livro, o premiado foi o capítulo 02, intitulado "VACINAS CONTRA COVID-19: UMA BREVE DESCRIÇÃO POR MEIO DE REVISÃO INTEGRATIVA“. 


\section{SUMÁRIO}

CAPÍTULO 1

A IMPORTÂNCIA E OS DESAFIOS DA ESTRATÉGIA DE SAÚdE DA FAMÍLIA NO ENFRENTAMENTO DA COVID-19

Romana Erica Tavares Grangeiro Pinto

Wyara Ferreira Melo

Maria Amanda Laurentino Freires

Patrício Borges Maracajá

Aline Carla de Medeiros

José Cândido da Silva Nóbrega

Manoel Marques de Souto Nóbrega Filho

Túlio Alberto de Oliveira Sousa

Mônica Valéria Barros Pereira

Vicente Saraiva dos Santos Neto

Hozanna Estrela Celeste

Gabriela Rocha Pordeus dos Santos

DOI: 10.47094/978-65-88958-41-4/17-26

CAPÍTULO 2

VACINAS CONTRA COVID-19: UMA BREVE DESCRIÇÃO POR MEIO DE REVISÃO INTEGRATIVA

Fernanda Lima Marçal

Isabela Figueiredo e Souza

Maria Eduarda Coelho Gomes

Larissa Lima Torres

Isabela Campbell Santos

Thamara Lóren Lima

Ludmilla Vieira Magalhães

Maria Eduarda Sirina Pereira

Lucas Viana de Oliveira 
Larissa da Silva Torres França

Natan Fiorotti da Silva

Milena de Oliveira Simões

DOI: 10.47094/978-65-88958-41-4/27-41

CAPÍTULO 3

AÇÃO EM SAÚdE E A IMPORTÂNCIA DO USO CORRETO DAS MÁSCARAS PARA PREVENÇÃO DE INFECÇÕES RESPIRATÓRIAS

Soniely Nunes de Melo

Rafael Belarmino de Souza Lima

Tarcísio Correia Sposito

Rayana Ribeiro Trajano de Assis

Nayara Sandriele Santana de Souza

Bruna Rafaella Santos Torres

Flávio José Alencar de Melo

Davi Silva de Jesus

Carlos Henrique Bezerra de Siqueira

Izabelle Barbosa da Silva

Marcos André de Holanda Prudente Pessoa

Ana Marlusia Alves Bomfim

DOI: 10.47094/978-65-88958-41-4/42-51

CAPÍTULO 4

CONHECIMENTOS DAS EQUIPES MULTIPROFISSIONAIS DA ESTRATÉGIA DE SAÚDE DA FAMIILIA SOBRE COVID-19 E GESTAÇÃO

Priscilla dos Santos Nascimento

Michelle Araújo Moreira

DOI: 10.47094/978-65-88958-41-4/52-65 
CARTILHA EDUCATIVA SOBRE A COVID-19 PARA A PROTEÇÃO DA COMUNIDADE ACADÊMICA DO CAMPUS BELÉM DO IFPA

Lidineusa Machado Araujo

Maria de Nazaré Pereira Rodrigue Martins

Gabriela Priscila de Lima Carvalho

Fernanda Rafaela de Souza Rebelo da Costa

Michelle da Silva Pereira

Andréa de Melo Valente

Maria Helena Cunha Oliveira

Antônio Marcos Mota Miranda

DOI: 10.47094/978-65-88958-41-4/66-75

CAPÍTULO 6 .76

A SAÚdE DOS PROFISSIONAIS DE SAÚDE QUE ATUAM NA LINHA DE FRENTE NA PANDEMIA DO COVID - 19: REVISÃO INTEGRATIVA

João Lucas Ferreira Andrade

Léa Bianch Lima

Luana Kellen Nogueira Epitácio

Maria Eduarda Alves Vasconcelos

Antônio Augusto Ferreira Carioca

Carlos Antônio Bruno da Silva

Eudóxia Sousa de Alencar

DOI: 10.47094/978-65-88958-41-4/76-89

CAPÍTULO 7 .90

OS EFEITOS DA PANDEMIA NA COMPULSÃO ALIMENTAR: UMA REVISÃO DE LITERATURA

Lais Pontes de Miranda Cerqueira

Tarcio Goncalves sobral

Isadora Bianco Cardoso de Menezes

DOI: 10.47094/978-65-88958-41-4/90-97 
O IMPACTO DA PANDEMIA DO COVID - 19 SOBRE A SEGURANÇA ALIMENTAR E NUTRICIONAL: REVISÃO INTEGRATIVA

Bruna Sousa Barbosa

Igor Matheus Cruz de Oliveira

João Lucas Ferreira Andrade

Léa Bianch Lima

Luana Kellen Nogueira Epitácio

Maria Eduarda Alves Vasconcelos

Antônio Augusto Ferreira Carioca

Carlos Antônio Bruno da Silva

Eudóxia Sousa de Alencar

DOI: 10.47094/978-65-88958-41-4/98-104

CAPÍTULO 9 105

A UTILIZAÇÃO DOS PROTOCOLOS DE USO DO GUIA ALIMENTAR PARA A POPULAÇÃO BRASILEIRA: RELATO DE EXPERIÊNCIA

Bárbara Santos Amorim

Lis Chaves Marinho

Isadora Bianco Cardoso de Menezes

DOI: 10.47094/978-65-88958-41-4/105-114

CAPÍTULO 10 .

O IMPACTO DA COVID-19 EM PORTADORES DE SÍNDROME METABÓLICA

Laura Rasul de Lima

Ana Beatriz Amaral Vieira

Gabriella Neiva Reis

Ingrid Ravenna Liberalino Lima

DOI: 10.47094/978-65-88958-41-4/115-122 
SIMPLIFICANDO A HANSENÍASE: PROJETO DE INTERVENÇÃO REALIZADO EM

MEIO DIGITAL DURANTE A PANDEMIA

Amanda Almeida Lima

Ana Beatriz Sousa Santos

Francisco Vittor Miranda e Araújo

Jesamar Correia Matos Filho

João Ferreira de Paula Neto

Maria Clara de Freita Albano

Manoel Cícero Viana de Lima

Pedro Schmitt Martins Paiva Matos

Ruddy Mariano Maia Cysne Guerra

Samuel Carvalho Vasconcelos

Thaine Mirla Rocha

Elaine Lopes Bomfim

DOI: 10.47094/978-65-88958-41-4/123-131

CAPÍTULO 12.

DESCOMPLICANDO A HANSENÍASE: PROJETO DE INTERVENÇÃO REALIZADO EM UNIDADE DE SAÚDE NO PERÍODO DA PANDEMIA

Adália Stefanny de Araujo Cavalcante

Giovanna Giffoni Souza do Nascimento

Iêda de Freitas Martins Jota

Isabel Camila Araújo Barroso

Kaio Rangel Freitas Guimarães

Laís Mesquita de Sousa

Monique dos Santos Chaves

Manoel Victor Freires Vieira

Matheus Macedo Braga Coelho

Thaine Mirla Rocha

Elaine Lopes Bomfim 
CAPÍTULO 13. 140

HANSENÍASE: UM ESTUDO EPIDEMIOLÓGICO NA REGIÃO DO MÉDIO ARAGUAIAMATO GROSSO

Flavia Rodrigues Santana

Josilene Dália Alves

DOI: 10.47094/978-65-88958-41-4/140-150

CAPÍTULO 14 151

MUNICÍPIOS COM ALTA INCIDÊNCIA DE TUBERCULOSE EM MATO GROSSO: CONHECER PARA INTERVIR EM TEMPOS DE PANDEMIA

Larissa Machado Bellé

Yasmim Paloma Abreu Silva

Alessandro Rolim Scholze

Josilene Dália Alves

DOI: 10.47094/978-65-88958-41-4/151-162

CAPÍTULO 15 163

SAÚDE INTEGRAL DA MULHER EM CONTEXTO PANDÊMICO: REVISÃO INTEGRATIVA DE LITERATURA

Lohana Guimarães Souza

Tailande Venceslau Carneiro

Letícia Grazielle Santos

DOI: 10.47094/978-65-88958-41-4/163-175

CAPÍTULO 16 176

ASSISTÊNCIA DE ENFERMAGEM NO OUTUBRO ROSA E PERFIL EPIDEMIOLÓGICO DAS MULHERES: RELATO DE EXPERIÊNCIA

Helena Pereira de Souza

Laura Letícia Perdição Guerra

Luana Fernandes e Silva 
Thales Philipe Rodrigues da Silva

Alessandra Lage Faria

Helen Carine Ferreira Balena

Érica Moreira de Souza

Bruna Luíza Soares Pinheiro

Lorena Medeiros de Almeida Mateus

Flávia Duarte de Oliveira Ribeito

Bianca Maria Oliveira Luvisaro

Fernanda Penido Matozinhos

DOI: 10.47094/978-65-88958-41-4/176-188

CAPÍTULO 17 189

AGRAVAMENTO DAS DOENÇAS PSIQUIÁTRICAS DURANTE O PERÍODO DE ISOLAMENTO SOCIAL: UMA BREVE REVISÃO DE LITERATURA

Hellen Kristina Magalhães Brito

Gabriela Teixeira Lima

Ana Laura Fernandes Tosta

Laura Beatriz Caitano de Oliveira

Maria Paula Ricardo Silva

Mariana Vieira Garcia de Carvalho

Nathália Siriano Costa

Mayara Rita Figueredo

Mabel Fernandes Rocha

Helena Maria Mendes Marques

Kaio Murilo Santana Corrêa

Ana Flávia Buiatte Andrade

DOI: 10.47094/978-65-88958-41-4/189-200 
ATUAÇÃO DO PSICÓLOGO EM TEMPO DE PANDEMIA: UMA REVISÃO DE ESTUDOS NACIONAIS

Gabriel Rigamonte

Sueli Souza

Wilson Quiroz

Daniel Bartholomeu

Fernando Pessotto

Cintia Heloína Bueno

Fernanda Helena Viana Garcia

DOI: 10.47094/978-65-88958-41-4/201-213

CAPÍTULO 19

CRIAÇÃO DE UM APLICATIVO VOLTADO PARA UMA UNIDADE BÁSICA DE SAÚdE EM FORTALEZA

Isabella Araujo Duarte

Giovanna Rolim Pinheiro Lima

Idna Lara Goes de Sena

Laura Figueiredo Leite

Letícia Cavalcante Lócio

Livian Araújo Camelo Gomes

Maria Regina Cardoso Linhares Oliveira Lima

Maria Tereza Linhares Cardoso

Pedro Henrique Cardoso Nogueira

Rafael Albuquerque Franco

Rodrigo Carvalho Paiva

Berta Augusta Faraday Sousa Pinheiro

DOI: 10.47094/978-65-88958-41-4/214-229 
TELEMEDICINA E SUAS VARIÁVEIS NO CENÁRIO DE PANDEMIA MUNDIAL: UMA REVISÃO DE LITERATURA

Raniere Canteiro Garcia Lhamas

Andressa Marcolino Campos

Douglas Ferreira Lima

Gabriel Souza Ferreira Oliveira

Guilherme de Mendonça Lopes Beltrão

Luciana de Paula Santana

Nícollas Nunes Rabelo

DOI: 10.47094/978-65-88958-41-4/230-237

CAPÍTULO 21 238

MONITORIA ACADÊMICA NO ENSINO REMOTO: PERSPECTIVAS E DESAFIOS EM TEMPOS PANDÊMICOS

Felipe Gabriel Assunção Cruz

Givanildo Bezerra de Oliveira

Marcílio Delan Baliza Fernandes

Ana Lúcia Moreno Amor

DOI: 10.47094/978-65-88958-41-4/238-248

CAPÍTULO 22

A DOENÇA DO OLHO SECO NA SÍINDROME DE SJÖGREN

Bruna Rafaella Santos Torres

Carlos Eduardo Ximenes da Cunha

Carlos Henrique Bezerra de Siqueira

Flavia Emanuelly Alves França Gomes

Santília Tavares Ribeiro de Castro e Silva

Anna Caroline Guimarães Gomes

Laís Rytholz Castro

Dennis Cavalcanti Ribeiro Filho 
Lara Medeiros Pirauá de Brito

Marina Viegas Rezende Ribeiro

DOI: 10.47094/978-65-88958-41-4/249-259

CAPÍTULO 23 260

FEIRAAGROECOLÓGICA: DIFICULDADES E POTENCIALIDADES DE UM CIRCUITO CURTO DE COMERCIALIZAÇÃO

Maria Rita Garcia de Medeiros

Rônisson Thomas de Oliveira Silva

Maria Natalícia de Lima

Ana Beatriz Macêdo Venâncio dos Santos

DOI: 10.47094/978-65-88958-41-4/260-269

CAPÍTULO 24 270

AVALIAÇÃO DO IMPACTO DE UMA REDE SOCIAL EM USUÁRIOS DE UMA UNIDADE BÁSICA DE SAÚDE EM FORTALEZA

Luiz Gerson Gonçalves Neto

Letícia Cavalcante Lócio

Carlos Alexandre Leite Pereira Filho

Henrique Sousa Costa

Maria Helena dos Santos Macedo

Lígia Bringel Olinda Alencar

Berta Augusta Faraday Sousa Pinheiro

Isaac Dantas Sales Pimentel

DOI: 10.47094/978-65-88958-41-4/270-280 


\section{Hellen Kristina Magalhães Brito ${ }^{1}$;}

Centro Universitário Atenas (UniAtenas), Paracatu, MG.

http://lattes.cnpq.br/2848475042427928

\section{Gabriela Teixeira Lima²;}

Centro Universitário Atenas (UniAtenas), Paracatu, MG.

http://lattes.cnpq.br/8715223474218660

Ana Laura Fernandes Tosta ${ }^{3}$;

Centro Universitário Atenas (UniAtenas), Paracatu, MG.

http://lattes.cnpq.br/6711193374939869

\section{Laura Beatriz Caitano de Oliveira ${ }^{4}$;}

Centro Universitário Atenas (UniAtenas), Paracatu, MG.

http://lattes.cnpq.br/5501024961785423

\section{Maria Paula Ricardo Silva ${ }^{5}$;}

Centro Universitário Atenas (UniAtenas), Paracatu, MG.

http://lattes.cnpq.br/3705183078365925

Mariana Vieira Garcia de Carvalho ${ }^{6}$;

Centro Universitário Atenas (UniAtenas), Paracatu, MG.

http://lattes.cnpq.br/9881315722628150

\section{Nathália Siriano $\operatorname{Costa}^{7}$;}

Centro Universitário Atenas (UniAtenas), Paracatu, MG.

http://lattes.cnpq.br/1295249715958968

\section{Mayara Rita Figueredo ${ }^{8}$;}

Centro Universitário Atenas (UniAtenas), Paracatu, MG.

https://orcid.org/0000-0002-3960-3699

\section{Mabel Fernandes Rocha';}

Centro Universitário Atenas (UniAtenas), Paracatu, MG. 


\section{Helena Maria Mendes Marques ${ }^{10}$;}

Centro Universitário Atenas (UniAtenas), Paracatu, MG.

http://lattes.cnpq.br/8791802446169089

\section{Kaio Murilo Santana Corrêa ${ }^{11}$;}

Centro Universitário Atenas (UniAtenas), Paracatu, MG.

http://lattes.cnpq.br/7840841582866214

\section{Ana Flávia Buiatte Andrade ${ }^{12}$.}

Centro Universitário Atenas (UniAtenas), Paracatu, MG.

https://orcid.org/0000-0002-7115-0650

RESUMO: A Organização das Nações Unidas alerta que o mundo passará por outra forte crise, dessa vez na saúde mental, como consequência da atual pandemia pelo COVID-19. Estudos qualitativos identificaram uma série de respostas psicológicas à quarentena, como confusão, medo, raiva, luto, dormência e insônia induzida por ansiedade. Além disso, com base em evidências científicas constatadas em epidemias anteriores, estima-se que distúrbios como transtorno de ansiedade generalizada, depressão e transtorno de estresse pós-traumático sejam ampliados significativamente. Viu-se que a política do isolamento, por mais benéfica que possa ser no controle da propagação da doença, ela pode ser responsável por desencadear tais distúrbios psicológicos, que por vezes podem apresentar-se de forma permanente. Ademais, a gama de informações contrastantes e de cunho trágico também corrobora para o grande impacto na saúde mental dessas pessoas. Isso, pois, alimenta ainda mais as sensações de medo, incertezas e insegurança com que o que está por vir. Com base, portanto, em eventos anteriores, foi possível averiguar que a terapêutica multidisciplinar possui significativo impacto positivo, tanto aos pacientes, quanto às equipes de saúde frente ao combate à pandemia. Este trabalho objetiva enumerar as repercussões sobre a saúde mental derivadas de longos períodos de isolamento social e propor medidas de intervenção capazes de minimizar as consequências.

PALAVRAS-CHAVE: Saúde Mental. COVID-19. Isolamento Social.

\section{AGGRAVATION OF PSYCHIATRIC DISEASES DURING THE PERIOD OF SOCIAL ISOLATION: A BRIEF LITERARY REVIEW}

ABSTRACT: The United Nations warns that the world will experience another major crisis, this one about mental health due to the current pandemic by COVID-19. Qualitative studies have identified several psychological responses to quarantine, such as confusion, fear, anger, grief, numbness, and anxiety-induced insomnia. Besides, based on scientific evidence from previous epidemics, it is estimated that disorders such as generalized anxiety disorder, depression, and post-traumatic stress disorder have significantly increased. It was seen that the policy of isolation, as beneficial as it may be 
in controlling the spread of the disease, can be responsible for triggering such psychological disorders, which can sometimes be permanent. Moreover, the range of contrasting and tragic information also contributes to the great impact on the mental health of these people. This, because it feeds even more the feelings of fear, uncertainty, and insecurity about what is to come. Based on previous events, it was possible to ascertain that multidisciplinary therapy has a significant positive impact, both on patients and on health care teams, when fighting the pandemic. This paper aims to enumerate the repercussions on mental health derived from long periods of social isolation and to propose intervention measures capable of minimizing the consequences.

KEY-WORDS: Mental Health. COVID-19. Social Isolation.

\section{INTRODUÇÃO}

O primeiro caso de pneumonia com etiologia desconhecida foi registrado em Wuhan, província de Hubei, na China, em dezembro de 2019. Os casos se disseminaram de forma exponencial, e finalmente o agente etiológico foi sequenciado, revelando um vírus da família Coronovidae, nomeado como novo coronavirus-19 e renomeado como SARS-CoV-2.

Devido à rapidez com que ocorre a disseminação do vírus e contaminação dos seres humanos (cada paciente é capaz de transmitir, em média, para mais 2,74 pessoas), em 11 de março de 2020 a Organização Mundial de Saúde (OMS) declarou estado de pandemia pela doença do novo coronavírus (COVID-19).

Sabe-se que a transmissão ocorre por meio de gotículas respiratórias através do ar ou pelo contato com secreções contaminadas, como: espirro, tosse, gotículas de saliva, aperto de mão, ou superfícies de objetos contaminados. Qualquer pessoa que esteja próxima - aproximadamente um metro - a outra contaminada está exposta à provável infecção.

Ao verificar os resultados positivos do isolamento social em Wuhan, foi proposto que a medida fosse reproduzida mundialmente, pois reduzir o contato físico entre as pessoas parecia uma medida de grade valia, considerando a sua forma de disseminação. Como o vírus possui um período de incubação de até 14 dias, estabeleceu-se que a população ficasse também por 14 dias em completo isolamento social, período designado por "quarentena".

A reclusão de indivíduos trata-se de uma política de extrema eficácia no que se refere à proliferação de patógenos e preservação da saúde, independente da etiologia. No entanto, cursa com repercussões em longo prazo extremamente complexas e, dentre elas, destacamos o prejuízo à saúde mental das pessoas submetidas ao estado de isolamento social.

Segundo a Organização Pan-Americana da Saúde (OPAS), uma pandemia de tal grandiosidade tem consequências psicossociais que podem ultrapassar a capacidade de enfrentamento da população acometida. Calcula-se que metade das pessoas expostas ao vírus possa sofrer algum sintoma psicopatológico, considerando a magnitude alcançada pela doença e o grau de vulnerabilidade individual. 
A exposição prolongada ao estresse provocado pela quarentena e ao excesso de informações de cunho negativo e por vezes discordantes - bombardeadas pela mídia são gatilhos para o adoecimento psicológico. O medo intensifica os níveis de estresse e ansiedade em pessoas aparentemente saudáveis, e ainda exacerba os transtornos psiquiátricos já existentes.

Atrelado à pandemia de COVID-19, surge um estado de pânico social em nível global. Vivenciar o isolamento social poderá despertar sentimentos como angustia, insegurança e medo, que têm potencial de permanecerem até mesmo após o controle da pandemia.

Diante desse contexto, o objetivo do presente estudo é enumerar as repercussões sobre a saúde mental derivadas de longos períodos de isolamento social, juntamente aos resultados já experimentados em eventos históricos semelhantes. Propõem-se também medidas de intervenção capazes de minimizar o impacto já prenunciado na saúde mental.

\section{METODOLOGIA}

Este trabalho possui uma abordagem qualitativa, de natureza básica. Trata-se de uma pesquisa bibliográfica descritiva e documental, realizada nas bases PubMed, Lilacs e SciELO, utilizandose os termos "pandemia", "COVID-19" e "Saúde Mental”. Foram incluídos artigos de revisão e estudos observacionais, publicados de 2004 a 2020, em língua portuguesa, espanhola e inglesa com acesso aberto. Foram excluídos artigos incompletos, que não tratassem do tema estudado e cartas ao editor. Optou-se por trabalhos que versassem sobre consequências inerentes aos longos períodos de isolamento, excesso de informações e incertezas sobre o futuro na saúde mental da população em meio à pandemia por COVID-19.

\section{RESULTADOS}

Foram selecionados 28 artigos para a elaboração dessa breve revisão de literatura. Buscou-se eleger trabalhos que retratassem, por meio de diferentes metodologias, as inúmeras consequências psicossociais em pessoas submetidas a longos períodos de distanciamento social. Estudos tangenciando outras epidemias, como a do Ebola na África Ocidental em 2014, a SARS no Canadá e na China em 2003 e a pandemia por H1N1 em 2009 também foram utilizados para a reflexão do aumento e da exacerbação de distúrbios como depressão, transtorno de ansiedade generalizada (TAG) e transtorno do estresse pós-traumático (TEPT).

Em 2004 no Canadá, a Associação Americana de Psiquiatria realizou um estudo com profissionais da saúde que tiveram contato com pacientes acometidos por SARS. Nessa ocasião, verificou-se que a quarentena foi o gatilho mais importante para o surgimento de sintomas de transtorno de estresse agudo. Nesse mesmo estudo, a equipe também apresentou forte tendência em declarar exaustão, distanciamento de outras pessoas, ansiedade ao lidar com pacientes em estado de febre, insônia, baixa concentração, irritabilidade, diminuição do desempenho no trabalho, hesitação em ir para o trabalho e alguns consideravam até demissão. 
Estudos realizados com sobreviventes de desastres observaram que cerca de $75 \%$ das pessoas que apresentavam TEPT imediatamente após o trauma permaneciam neste estado por aproximadamente um ano. Outro trabalho constatou uma taxa de persistência de $40 \%$ para elevados níveis de TEPT entre profissionais da saúde, mesmo três anos após o surto de SARS em países asiáticos e no Canadá. Estudos apontam que, se os sintomas de TEPT perdurarem por mais de seis meses após o evento estressor, é presumível que persista também em longo prazo.

Em um estudo de coorte, também realizado para avaliar os efeitos psicológicos na população que sofreu o surto de SARS em 2003, participaram 1057 pessoas. Nesse trabalho foi possível constatar que as pessoas que se encontravam em isolamento com outras potencialmente contaminadas por coronavírus, referiam diversas respostas negativas no período da quarentena. Das 1057 pessoas estudadas, mais de $20 \%$, relataram medo, $18 \%$ tristeza, $18 \%$ nervosismo e $10 \%$ relataram sentir culpa. Poucos foram os que relataram sentimentos positivos como felicidade, representando $5 \%$ do grupo pesquisado, e alívio em apenas 4\%. Estudos qualitativos também identificaram uma série de outras respostas psicológicas à quarentena, como confusão, raiva, luto, dormência e insônia induzida por ansiedade.

Recentemente a China realizou um estudo transversal, no qual participaram 1257 profissionais da saúde que trataram de pacientes com COVID-19. Na ocasião foi apontada alta prevalência de sintomas de desequilíbrio da saúde mental desses profissionais. O estudo revelou que 50,4\% dos pesquisados queixou-se de sintomas depressivos, 44,6\% relatou ansiedade, $34 \%$ passou a sofrer de insônia e 71,5\% referiram angústia. Embora esses achados sejam alarmantes, não são independentes. A pesquisa realizada sobre o efeito psicológico de surtos mundiais por doenças infecciosas demonstrou associações entre ansiedade relacionada à pandemia e sintomas elevados de estresse, ansiedade, preocupações com contaminação, estresse pós-traumático e suicídio.

Outro trabalho também realizado na China em janeiro de 2020, mas agora com a população em geral, contou com uma amostra de 1210 participantes em 194 diferentes cidades chinesas, onde foi aplicado um formulário online para pesquisa. Nesse estudo, buscou-se avaliar as repercussões psicológicas, níveis de ansiedade, estresse e depressão nos estágios iniciais da pandemia por COVID-19. Constatou-se que 53,8\% dos participantes classificaram o impacto psicológico da pandemia como moderado ou grave; $16,5 \%$ declararam sintomas depressivos moderados ou graves; $28,8 \%$ queixaram sintomas de ansiedade moderados ou graves; e ainda $8,1 \%$ relataram níveis de estresse moderado ou grave. Os autores ainda frisaram que $84,7 \%$ dos entrevistados, passou de 20 a 24 horas por dia dentro de suas casas; $75,2 \%$ demostraram preocupação que os familiares contraíssem o COVID-19; e ainda que $75,1 \%$ consideravam as informações relacionadas à saúde satisfatórias.

Professores vinculados à Universidade do Estado do Rio de Janeiro (UERJ) publicaram recentemente, na revista científica The Lancet, um estudo referente aos impactos da pandemia pelo COVID-19 na saúde mental da população em geral. Nesse cenário, constatou-se que houve um aumento alarmante de $90 \%$ nos casos de depressão e, no que diz respeito à ansiedade, os casos mais que duplicaram. Além disso, o estudo também apontou significativo aumento em outras manifestações psicossociais advindas do isolamento, como exaustão, solidão, irritabilidade, insônia, tédio, falta de 
concentração, desapego de outras pessoas e indecisão.

Com o objetivo de mensurar o impacto que a atual situação ocasionada pelo coronavírus afeta a saúde mental da população, uma pesquisa online chinesa foi realizada com 1577 pessoas e 214 profissionais de saúde. Os resultados revelaram que um quinto das pessoas não vinculadas à área de saúde demonstraram sinais de depressão e ansiedade, sendo a mesma proporção observada entre os profissionais de saúde. $\mathrm{Na}$ análise, foram selecionadas pessoas não infectadas pelo coronavírus, mas que tiveram contato próximo com alguém contaminado e ainda que despendiam um tempo maior ou igual a duas horas diárias pesquisando por notícias sobre o coronavírus nas redes sociais. $\mathrm{O}$ estudo ainda mostrou que o apoio social diminui os sintomas de ansiedade e depressão. A pesquisa incluiu a importância da internet no momento de isolamento social, principalmente com consultas e aconselhamentos online, porém recomendou cautela ao uso excessivo para informações sobre a pandemia.

Em 1918 surgiu uma das pandemias mais devastadoras que a humanidade já vivenciou. Identificada como H1N1, mais conhecida como "gripe espanhola", ela foi considerada de altíssima periculosidade clínica. Tanto é que causou entre 50 a 100 milhões de óbitos no mundo inteiro.

Após a gripe espanhola, o mundo testemunhou diversas outras pandemias. Dentre elas destacamos a gripe asiática de 1957, causada pelo vírus H2N2, cuja mortalidade foi inferior $0,1 \%$ em razão do melhor desenvolvimento na área da saúde. Posteriormente, houve a pandemia de Hong Kong em 1968, causada pelo vírus H3N2, felizmente menos alarmante que as anteriores.

Em 2003, países asiáticos e o Canadá vivenciaram um surto de Síndrome Respiratória Aguda (SARS), o qual exigiu também medidas de isolamento, de modo semelhante ao que vivenciamos hoje com a pandemia pelo Sars-CoV-2, em que todas regiões expostas foram colocadas em quarentena durante a crise. E ainda em 2014 na África Ocidental, vilas inteiras vivenciaram o isolamento durante o surto do Ebola.

Em 2009 o mundo vivenciou a pandemia causada pelo H1N1, responsável por uma alta taxa de mortalidade, mas com menor capacidade de transmissão. Durante esse acontecimento, constatou-se que dentre os pacientes internados em unidades de tratamento intensivo (UTIs), 50\% desenvolveram ansiedade, $25 \%$ depressão e $40 \%$ apresentaram risco para desenvolverem transtorno pós-traumático.

\section{DISCUSSÃO}

Desde o início do século $\mathrm{XX}$, o mundo todo tem sido posto à prova com o surgimento de diversas doenças infeciosas com altos níveis de gravidade e letalidade. Por mais que a medicina e a área cientifica de modo geral tenham passado por vultosa evolução, nenhum país ainda está preparado para lidar com doenças altamente contagiosas. Por este motivo é que existe uma enorme urgência em compreender quais as possíveis repercussões psicossociais após uma patologia de tão rápida disseminação. 
Diante de tantas evidências, o apelo para que a população fique em casa se destaca em meio às estratégias propostas para enfrentamento da pandemia do novo coronavírus, com o objetivo de diminuir sua transmissão. Em contrapartida, a tendência é que os profissionais de saúde mantenham ou aumentem sua jornada de trabalho, passando longos períodos exposto ao COVID-19 diariamente.

Muitos desses profissionais que atuam na linha de frente do combate à pandemia foram infectados em todo o mundo. Na Itália, esse número chegou a $20 \%$ no final do mês de março de 2020, mesmo com uso correto de equipamentos para proteção individual (EPI's). Portanto, não basta somente ter acesso aos EPI's, mas é necessário que esses sejam eficientes e de alta qualidade, condição que se tornou a preocupação central para esses trabalhadores.

É fato que o distanciamento social é indispensável para a contenção da disseminação de doenças infecciosas. Porém, a quarentena é uma vivência extremamente desgastante para quem precisa ser submetido a ela. É um período em que os entes queridos são obrigados a se manterem distantes, a liberdade é extinta, não é permitido reunir-se com amigos aos finais de semana, nem mesmo visitar entes queridos ou até os que estão enfermos. E, caso alguém venha a falecer, não se autorizam velórios, por tratar-se de aglomeração de pessoas com potencial risco de contaminação.

Além disso, tem-se ainda a incerteza sobre o status da doença; a quantidade de notícias negativas persistentes, por vezes até conflitantes; e a insegurança, não só da população em geral, mas principalmente dos trabalhadores da área da saúde em se contaminarem, ou acabar contaminando àqueles que mais querem cuidar. Centenas de sentimentos e sensações que juntos se tornam extremamente danosas à integralidade da saúde mental desses indivíduos.

Diante de tanta evidência, é possível presumir que uma das piores crises em saúde mental ainda está por vir. Por mais semelhante que possam ter sido as outras pandemias já testemunhadas pela humanidade, nenhuma delas ocorreu na proporção que o COVID-19 tem tomado. O período de isolamento social já ultrapassou qualquer precedente histórico, além do número de pessoas contaminadas e óbitos já registrados serem os maiores já descritos. Nenhum continente saiu ileso dessa infeção tão devastadora. E por mais recente que seja, há diversos estudos que também atestam acerca da plenitude psicológica que tem sido tão violentamente lesada.

Cabe ainda ressaltar que o impacto da pandemia na saúde mental também ocorre naqueles indivíduos que não foram diretamente afetados pelo COVID-19. Dois fatores desencadeantes consistem na sobrecarga de preocupação e no acúmulo de função gerados por mudanças do próprio cotidiano, como os referentes ao trabalho, tarefas domésticas, alimentação, transporte, economia, educação, entre outros. Além disso, o medo do contágio e do adoecimento soma-se com outras questões intrínsecas a cada indivíduo, e ainda tem-se o consumo excessivo de informações como um novo fator de contribuição significativa para a instalação desse cenário.

O físico espanhol Alfons Cornella em 1996 definiu este quadro pela primeira vez, dando-lhe o nome de "infoxicação", um neologismo entre os termos informação e intoxicação. Diante do cenário atual, a OMS usa o termo "infodemia", definido como um excesso de informações que podem ou não ser precisas e confiáveis. Tais referências errôneas são capazes de aumentar o sofrimento por antecipação. O próprio Diretor Geral da OMS, Tedros A. Ghebreyesus fez menção a este fenômeno 
em 15 de fevereiro de 2020, quando o COVID-19 ainda era designado como epidemia.

Diante de tanto malefício resultante da pandemia pelo COVID-19 e do processo de isolamento social atrelado a ela, é de extrema importância que haja medidas de intervenção capazes de minimizar os efeitos psicossociais já esperados para população. O psicólogo Zacarias Ramalho afirma que uma forma de escapar desses sentimentos ruins é controlando o que se consome de informações. Declara ainda que o melhor é analisar o número de informações a que se tem acesso e, sobretudo, fazê-lo por fontes confiáveis. Zacarias recomenda dispor um único momento do dia para se informar com notícias que auxiliarão neste cenário. Desligar-se completamente de todas as fontes de informação não é uma alternativa, uma vez que as orientações dos órgãos de saúde e atualizações sobre a situação atual do país são fundamentais durante este período de pandemia. A busca pelo equilíbrio é o ideal para esse momento. Outro fator que contribui positivamente neste quesito é dedicar parte do dia a atividades de lazer, como ler livros e assistir filmes, sendo ainda de extrema importância dedicar esse tempo para um momento de planejamento.

Outra proposta para o novo contexto de saúde mental é a psiquiatria digital e suas ferramentas, como inteligência artificial, telepsiquiatria e serviços de saúde mental assistidos por computadores, serviços autorizados pelos órgãos reguladores como o governo federal e os conselhos de cada profissão, após a conjuntura de pandemia. É de extrema importância que todos os serviços se adaptem em prol do auxílio ao combate dos danos advindos do coronavirus.

No Brasil, em 26 de março de 2020, foi publicada a Resolução CFP no 4/2020, que permite a prestação de serviços psicológicos por meios de tecnologia da informação e da comunicação após realização do "Cadastro e-Psi" pelo profissional, embora não seja necessário aguardar a emissão de parecer do respectivo Conselho Regional de Psiquiatria (CRP) para iniciar o trabalho remoto. Tal resolução suspende, durante o período de pandemia do COVID-19, os Art. $3^{\circ}, 4^{\circ}, 6^{\circ}, 7^{\circ}$ e $8^{\circ}$ da Resolução CFP n ${ }^{\circ} 11 / 2018$, que afirmam, respectivamente, a necessidade de um cadastro prévio junto ao CRP e sua autorização; a aplicação de falta disciplinar ao profissional que mantiver serviços psicológicos por meios tecnológicos de comunicação à distância sem o cadastramento no CRP; a inadequação dos atendimentos de pessoas e grupos em situação de urgência e emergência pelos meios de tecnologia e informação; o veto ao atendimento de pessoas e grupos em situação de emergência e desastres pelos meios de tecnologia e informação; e o veto ao atendimento de pessoas e grupos em situação de violação de direitos ou de violência pelos meios de tecnologia e informação. Sendo assim, passa a ser autorizada a prestação de serviços psicológicos por meios de tecnologia da informação e da comunicação às pessoas e grupos em situação de urgência, emergência e desastre, bem como violação de direitos ou violência, buscando minimizar as implicações psicológicas diante da COVID-19.

As intervenções voltadas à população geral incluem propostas psicoeducativas, tais como: cartilhas e outros materiais informativos; oferta de canais para escuta psicológica, de modo que as pessoas possam aliviar suas pressões negativas por via telefônica ou por atendimentos em plataformas online; ou atendimentos presenciais, quando comprovadamente necessários. Levantamentos online também têm sido realizados para melhor compreender o estado de saúde mental da população diante do COVID-19, com o objetivo de identificar rapidamente casos com maior risco e ofertar intervenções 
psicológicas alinhadas às demandas.

Sugere-se também, mesmo que de forma remota, oferta de cuidados psicológicos iniciais, como assistência humanizada e ajuda básica em situações de crise, ambas possíveis com o treinamento de profissionais (psicólogos, psiquiatras, assistentes sociais) e a elaboração de politicas públicas eficientes que minimizem os impactos econômicos e sociais da pandemia, buscando assim aliviar preocupações e gerar um maior conforto, ativar a rede de apoio social e das necessidades básicas, como acesso a água, alimentação e informação. Neste contexto, as intervenções psicológicas devem ser dinâmicas e focadas, primeiramente, nos estressores relacionados à doença ou às outras dificuldades enfrentadas durante esse período de isolamento social.

O apoio social é um importante aliado para diminuição de sintomas de ansiedade e depressão na comunidade e nos profissionais de saúde, pois o apoio emocional coopera substancialmente com o bem-estar desses indivíduos. Sendo assim, o uso da internet nesse momento é extremamente valioso, tanto para teleconsultas, minimizando a exposição dos pacientes a clínicas e hospitais, quanto para criação de novos hábitos e rotinas diárias, como: realizar atividades físicas, executar o oficio laboral em casa, manter o contato com os entes queridos ou aprender novas habilidades.

Além disso, a OMS recomenda a integração da saúde mental nos serviços de atenção básica. No Brasil, os atendimentos gratuitos a pessoas com desordens psicológicas são prestados nos Centros de Atenção Psicossocial (CAPS), na atenção primária a saúde. Nesse ambiente, encontramse profissionais especializados em saúde mental, como psiquiatras e psicólogos. Entretanto, não são todos os municípios brasileiros os abrangidos pelos CAPSs, o que transfere esse trabalho aos profissionais das Unidades Básicas de Saúde, muitas vezes composta apenas pelo médico generalista.

Além da ampliação do CAPS para agregar todos os municípios, torna-se essencial que os profissionais sejam capacitados para lidar com os efeitos pós-pandemia como ocorreu em Serra Leoa, onde houve surtos de Ebola. Enfermeiros da saúde pública receberam treinamento específico voltado para identificação e tratamento de pacientes que apresentassem algum sintoma de natureza psíquica.

Vale ressaltar que o estresse e as sequelas psiquiátricas entre os trabalhadores da linha de frente do combate ao coronavirus requerem atenção e reparação categóricas e imediatas. Isso inclui zelar pela prevenção da contaminação desses profissionais, assegurando equipamentos de proteção individual apropriados, oferecer atendimentos por psicólogos e/ou psiquiátricas com o intuito de atenuar os sintomas de ansiedade e fortalecer o apoio psicossocial.

Ademais, fomentar a comunicação entre as equipes de saúde, criando um ambiente de reciprocidade e cooperação empática, possibilita a evasão de sentimentos muitas vezes danosos, como o esgotamento e a exaustão emocional. É importante também alertá-los acerca dos sintomas de TEPT, ansiedade e depressão, para que possam identificar de forma precoce e buscar pelo tratamento adequado, tanto para si, quanto para a equipe e os pacientes. 


\section{CONCLUSÃO}

Esse estudo reitera a constatação dos prejuízos à saúde física e mental ocasionados por longos períodos de isolamento social. Fomenta a necessidade de cuidados à integralidade psicológica das pessoas no período pandêmico e pôs- pandêmico, possibilitando ações incisivas nas intervenções necessárias para conter o agravamento das patologias mentais. Defende ainda que o estabelecimento e a melhoria de condições que suprimem a sobrecarga emocional são de extrema urgência, sendo necessárias, primordialmente, na atenção básica ao indivíduo e aos profissionais de saúde, a fim de reduzir os danos psicossociais de forma ampla e eficaz.

\section{DECLARAÇÃO DE INTERESSES}

Nós, autores deste artigo, declaramos que não possuímos conflitos de interesses de ordem financeira, comercial, político, acadêmico e pessoal.

\section{REFERÊNCIAS}

AQUINO, E. M. L. et al. Social distancing measures to control the COVID-19 pandemic: Potential impacts and challenges in Brazil. Ciencia e Saude Coletiva, v. 25, p. 2423-2446, 2020.

ARROYO-SÁNCHEZ, A. S.; PAREDES, J. E. C.; VALLEJOS, M. P. C. Infodemia, la otra pandemia durante COVID-19. 2020.

BAI, Y. M. et al. Survey of stress reactions among health care workers involved with the SARS outbreak. Psychiatric Services, v. 55, n. 9, p. 1055-1057, 2004.

BROOKS, S. K. et al. The psychological impact of quarantine and how to reduce it: rapid review of the evidence. The Lancet, v. 395, n. 10227, p. 912-920, 2020.

ĆOSIĆ, K. et al. Impact of human disasters and Covid-19 pandemic on mental health: Potential of digital psychiatry. Psychiatria Danubina, v. 32, n. 1, p. 25-31, 2020.

FAGUNDES, S. M. G. et al. POLÍTICAS PÚBLICAS \& COVID-19 PET-PP ( PROGRAMA DE EDUCAÇÃO TUTORIAL EM POLÍTICAS PÚBLICAS ) - UTFPR Covid-19 e saúde mental : subproduto de uma crise pandêmica. p. 1-8, 2020.

FARO, A. et al. COVID-19 e saúde mental: a emergência do cuidado. Estudos de Psicologia (Campinas), v. 37, 2020.

JALLOH, M. F. et al. Impact of Ebola experiences and risk perceptions on mental health in Sierra Leone, July 2015. BMJ Global Health, v. 3, n. 2, p. e000471, 17 mar. 2018.

KALIL, I.; SANTINI, R. M. Coronavírus: pandemia, infodemia e política. Fespsp / Uerj, p. 21, 2020. LAI, J. et al. Factors Associated With Mental Health Outcomes Among Health Care Workers Exposed to Coronavirus Disease 2019. JAMA network open, v. 3, n. 3, p. e203976, 2020. 
LEE, S. A. Coronavirus Anxiety Scale: A brief mental health screener for COVID-19 related anxiety. Death Studies, v. 44, n. 7, p. 393-401, 2020.

LIMA, C. M. A. DE O. Information about the new coronavirus disease (COVID-19). Radiologia Brasileira, v. 53, n. 2, p. v-vi, 2020.

MEDEIROS, A. Y. B. B. V. et al. Fases psicológicas e sentido da vida em tempos de isolamento social pela pandemia de COVID-19 uma reflexão a luz de Viktor Frankl. Research, Society and Development, v. 9, p. 1689-1699, 2020.

MINISTÉRIO DA SAÚDE. FUNDAÇÃO OSWALDO CRUZ. PLANO DE CONTIGÊNCIA DA FIOCRUZ DIANTE DA PANDEMIA DA DOENÇA PELO SARS-CoV-2 ( COVID-19). Disponível em: <https://www.arca.fiocruz.br/bitstream/icict/40335/6/plano de contingencia_covid19_ fiocruzv1.0.pdf $>$.

NI, M. Y. et al. Mental Health, Risk Factors, and Social Media Use During the COVID-19 Epidemic and Cordon Sanitaire Among the Community and Health Professionals in Wuhan, China: CrossSectional Survey. JMIR mental health, v. 7, 2020.

OPAS. Policy Brief: COVID-19 and the Need for Action on Mental Health EXECUTIVE SUMMARY : COVID-19 and the Need for Action on Mental Health. World Health Organization, Geneva, swithzerland, p. 3-10, 2020.

ORNELL, F. et al. The impact of the COVID-19 pandemic on the mental health of healthcare professionals. Cadernos de saude publica, v. 36, n. 4, p. e00063520, 2020.

PEREIRA, M. D. et al. A pandemia deCOVID-19, o isolamento social, consequências na saúde mentale estratégias de enfrentamento: uma revisão integrativa. [s.d.].

PFEFFERBAUM, B.; NORTH, C. S. Mental Health and the Covid-19 Pandemic. New England Journal of Medicine, p. 1-2, 2020.

REYNOLDS, D. L. et al. Understanding, compliance and psychological impact of the SARS quarantine experience. Epidemiology and Infection, v. 136, n. 7, p. 997-1007, 2008.

SANTOS, I. A. DOS; NASCIMENTO, W. F. DO. As medidas de quarentena humana na saúde pública: aspectos bioéticos. Revista Bioethikos, v. 8, n. 2, p. 174-185, 2014.

SARTÓRIO, C. L. et al. Paradoxos de Retroalimentação da Pandemia da COVID-19 : quebrando o ciclo. Cadernos de Prospecção - Salvador, v. 13, p. 424-440, [s.d.].

SCHMIDT, B. et al. Impactos na Saúde Mental e Intervenções Psicológicas Diante da Pandemia do Novo Coronavírus (COVID-19) 1 Impacts on Mental Health and Psychological Interventions related to the New Coronavirus Pandemic (COVID-19) Lucas NEIVA-SILVA 5. p. 1-13, 2020.

SPRANG, G. Vicarious stress: patterns of disturbance and use of mental health services by those indirectly affected by the Oklahoma City bombing. Psychol Rep, v. 89(2), p. 331-338, 2001. 
WANG, C. et al. Immediate psychological responses and associated factors during the initial stage of the 2019 coronavirus disease (COVID-19) epidemic among the general population in China. International Journal of Environmental Research and Public Health, v. 17, n. 5, 2020.

WEISSMANN, L. et al. INFORME DA SOCIEDADE BRASILEIRA DE INFECTOLOGIA (SBI) SOBRE O NOVO CORONAVÍRUS. Sociedade Brasileira de Infectologia, n. 11, p. 4, 2020.

WU P et al. The psychological impact of the SARS epidemic on hospital employees in China: Exposure, risk perception, and altruistic acceptance of risk. Canadian Journal of Psychiatry, v. 54, n. 5, p. 302-311, 2009.

ZANIELLI, D. Quais as principais consequências metabólicas da pandemia por COVID-19? Disponível em: <https://pebmed.com.br/quais-as-principais-consequencias-metabolicas-dapandemia-por-covid-19/\%3E $>$. 


\section{Índice Remissivo}

$\mathbf{A}$

Acesso aos psicólogos 201, 203, 204

Acesso às redes sociais 43

Agricultor e o consumidor 260

Agricultores familiares 260, 263, 266

Alterações do metabolismo 115

Ambiente virtual 215

Ansiedade 77, 78, 81, 82, 83, 84, 86, 87, 91, 92, 94, 95, 117, 121, 190, 192, 193, 194, 197, 204, 207, 208, 209

Aplicativo 136, 215, 217, 218, 219, 220, 221, 222, 223, 224, 225, 226, 227, 228, 243, 271, 272, 273, 274, 278, 280

Aprendizagem 239

Assistência social 163, 171, 205

Associação da covid-19 com a gestação 52

Astrazeneca (universidade de oxford) 28, 30

Atenção primária 25, 124

Atenção primária à saúde (aps) 19, 59, 107, 125, 177, 178

Atendimento ambulatorial 105

Atendimento on-line 201, 203

Atendimento remoto 202, 205

Atividades econômicas 28

Autoanticorpos 250

Autoridades sanitárias 67, 69, 73, 278

C

Capacidade de defesa do organismo 115

Características dos imunizantes 28, 30

Cartilha educativa sobre a covid-19 67, 69, 70

Ceratoconjuntivite sicca $(\mathrm{kcs}) 250$

Combate à hanseníase 133

Comercialização 38, 260, 261, 262, 263, 264, 265, 266, 267, 268, 269

Compulsão alimentar 90, 92, 93, 94, 95, 96

Consumo de alimentos 105

Contaminação 43, 44, 45, 47, 49, 72, 73, 74, 78, 117, 135, 179, 191, 193, 195, 197

Coronavac (sinovac) 28, 29

Cuidados individuais e coletivos $67,69,73$

Cuidados preventivos ao covid-19 115

D

Depressão 78, 81, 82, 83, 87, 91, 92, 94, 95, 110, 172, 190, 192, 193, 194, 197, 207, 208, 209

Desemprego 21, 99, 103, 153

Desenvolvimento do câncer 177, 185

Desigualdade em saúde 163

Desinformação 29, 208 
Diabetes 21, 56, 57, 95, 105, 106, 107, 108, 114, 116, 117, 118, 119, 120, 181, 183, 186

Direito humano à alimentação adequada 103, 260

Disseminação da informação 67,73

Distanciamento social 25, 48, 55, 61, 68, 73, 90, 95, 126, 130, 192, 195, 202, 279

Distúrbios psicológicos 190

Doença infecciosa 53, 125, 133, 134, 140, 141, 151, 152

Doença multifatorial 250

Doenças 21, 24, 28, 29, 43, 54, 56, 57, 59, 69, 91, 95, 106, 107, 108, 115, 116, 117, 119, 120, 129, 133, 137, 140, 142, $149,161,163,164,167,171,172,173,177,185,186,193,194,195,207,210,217,222,223,227,250,271$, $272,273,278,279$

Doenças crônicas 105, 171, 218, 221, 227, 273

Doenças negligenciadas 140, 142

\section{$\mathbf{E}$}

Educação em saúde 19, 24, 43, 46, 48, 50, 67, 69, 73, 105, 108, 133, 134, 135, 136, 137, 145, 179, 185, 215, 216, 217, $227,228,238,240,248,271,272,274$

Efeitos colaterais $28,30,31,32,33$

Enfermagem 50, 56, 57, 58, 60, 61, 88, 89, 122, 149, 161, 176, 177, 178, 180, 188, 227, 240, 280

Enfermidade epidêmica 238

Enfrentamento da covid-19 18, 19, 20, 23, 24, 25

Epidemiologia 114, 140, 148, 149, 160, 161, 242, 248

Equipamentos de proteção 24, 43, 47, 78, 171, 197

Equipes multiprofissionais $52,54,55,56,59,60,61,62$

Estratégia saúde da família (esf) 18, 19, 23, 54

Estratégias nutricionais 105

Estresse 77, 78, 81, 82, 83, 86, 87, 88, 89, 94, 172, 190, 192, 193, 197, 206, 207, 209, 210, 211

Etiologia autoimune 250

Evidências científicas 29, 52, 56, 57, 59, 61, 62, 63, 76, 79, 99, 101, 190

F

Feiras agroecológicas 260, 261, 264

Ferramenta tecnológica de saúde 215

Fortalecimento da saúde púbica 67

Frequência cardíaca 230

G

Gestação 52, 53, 54, 57, 58, 59, 61, 62, 63, 224

Glândulas exócrinas 250, 251, 252, 253

Guia alimentar 105, 107, 108, 112, 113

$\mathbf{H}$

Hábitos alimentares 90, 92, 94, 95, 111, 112

Hanseníase 124, 125, 126, 127, 128, 130, 131, 133, 134, 135, 136, 137, 138, 139, 140, 141, 142, 143, 144, 145, 146, $147,148,149$

Herança genética 115

Hipertensão 57, 95, 105, 107, 108, 114, 115, 116, 118, 119, 120, 121, 181, 183, 186, 228, 229, 274, 276,278 
Hiposecreção lacrimal 250

I

Idosos 19, 21, 23, 54, 105, 108, 134, 137

Impacto da pandemia 99

Imunização 28, 29, 30, 35, 38, 179, 183, 186

Inclusão 20, 22, 52, 55, 56, 58, 75, 77, 79, 101, 163, 165, 167, 215, 226, 268

Infecções 28, 29, 38, 43, 44, 48, 57, 59, 60, 78, 165, 172, 180, 227, 251

Infecções respiratórias virais $43,44,48$

Informação de qualidade 271, 279

Informação em saúde 67,69

Informação sobre a hanseníase 124

Instabilidade econômica 99, 103

Instrumento de prevenção 43

Intervenção psicológica 202

Isolamento $23,48,55,60,62,81,90,91,92,94,100,142,164,177,179,190,191,192,193,194,195,196,197,198$, $199,203,204,205,207,208,210,242,261$

Isolamento social 23, 26, 62, 90, 92, 94, 100, 142, 164, 177, 179, 190, 191, 192, 194, 195, 196, 197, 198, 203, 205, 207, 208, 210, 242

$\mathbf{J}$

Janssen (johnson \& johnson) 28, 29, 30

$\mathbf{L}$

Lesões cutâneas 140, 143

Linha de frente 76, 78, 79, 81, 84, 86, 87, 88, 171, 195, 197, 202, 206, 207, 208, 210

M

Má qualidade do sono 77,87

Máscaras faciais 43

Material digital 43, 46

Medição biométrica 230

Medidas de isolamento 26, 28, 194

Medidores de glicose 230

Metodologia pico (problema, intervenção, contexto, resultado) 76, 79, 99, 101

Monitores do centro de ciências da saúde (ccs) 238

Monitoria 238, 239, 240, 241, 242, 243, 244, 246

Morbidade 115, 119

Mortalidade materna 163, 171, 172

Mudanças nos estilos de vida 90, 92, 93

Mycobacterium leprae 135, 140, 141, 146

N

Níveis socioeconômicos 43, 46

Novas modalidades de interações 238

Novo coronavírus sars-cov-2 18, 19 
Olho seco 250, 251, 252, 253, 254, 255, 256, 257, 258

Organização mundial de saúde (oms) 19, 52, 53, 54, 68, 92, 191

Organização pan-americana de saúde (opas) 52, 54

Órgãos oficiais de saúde 238, 247

Outubro rosa $177,178,179,184,185$

$\mathbf{P}$

Padrões dietéticos 115

Pandemia 18, 19, 20, 21, 23, 24, 25, 26, 28, 31, 38, 45, 47, 48, 53, 55, 68, 69, 73, 75, 76, 78, 79, 81, 82, 84, 86, 87, 88, $89,90,91,92,93,94,95,96,99,100,101,102,103,104,117,118,119,120,124,126,130,131,133,135,136$, $137,142,152,153,160,161,162,163,164,165,168,169,170,171,172,173,174,177,179,180,186,187$, 190, 191, 192, 193, 194, 195, 196, 197, 198, 199, 200, 203, 204, 205, 206, 207, 208, 210, 211, 212, 213, 238, $239,240,241,242,244,247,248,271,272,279,280$

Perfil alimentar 105

Perfil clínico 160, 177, 183, 185

Pfizer (pfizer e biontech) 28, 30

Políticas públicas de saúde 18, 24, 173, 204

Portadores de síndrome metabólica 115

Prática oftalmológica 250

Práticas do autocuidado 178,186

Pressão arterial 115,230

Prevenção 29, 33, 72, 170, 187, 221

Primeiros socorros 215, 218, 219, 225, 227, 273

Princípio ativo 28, 30, 33

Produção de alimentos 260

Profissionais de psicologia 202

Profissionais de saúde 18, 22, 24, 54, 56, 76, 78, 79, 81, 82, 84, 85, 86, 87, 89, 107, 125, 138, 151, 160, 170, 185, 194, $195,197,198,202,206,207,208,238$

Programa nacional de alimentação escolar (pnae) 99, 265

Promoção em saúde 43, 45, 271, 278

Propagação do vírus $43,47,48,67,69$

Q

Qualidade de vida 22, 47, 82, 106, 112, 155, 217, 226, 244, 250, 251, 254, 272, 278

Quarentena 90, 100, 121, 126, 170, 172, 190, 191, 192, 193, 194, 195, 199, 202, 206

$\mathbf{R}$

Recurso tecnológico 230

Redes sociais 43, 45, 46, 49, 133, 137, 194, 271, 272, 274, 278, 279

Responsabilidade individual e coletiva 18, 24

Restrição social 90, 92, 93 
Sars-cov- $20,21,29,30,31,32,33,34,35,38,39,40,45,49,53,56,57,58,59,60,65,88,120,163,164,165,168$, $173,191,199,238,239,240,242,243,247,248,279$

Saúde da família 18, 19, 20, 22, 23, 24, 25, 26, 52, 54, 55, 62, 64, 125, 135, 179, 187

Saúde da mulher 163, 166, 173

Saúde de maneira remota 133

Saúde dos profissionais $76,79,81,86,89$

Saúde dos trabalhadores $77,86,87$

Saúde integral 52, 163, 165, 167, 171

Saúde mental 78, 83, 88, 90, 93, 94, 95, 96, 172, 179, 190, 191, 192, 193, 194, 195, 196, 197, 198, 202, 203, 204, 206, $207,208,210,212,222,227,279$

Saúde psicológica 77,87

Saúde pública $6,20,28,38,69,73,82,120,125,134,139,141,148,149,160,168,169,170,171,172,177,179,197$, $199,208,211,238,240,242,247$

Secura ocular 250, 251, 257

Sedentarismo 94, 107, 115, 179

Segurança alimentar e nutricional 99, 100, 101, 102, 103, 104

Segurança alimentar nutricional (san) 99

Serviço de saúde 71, 115, 120, 180

Síndrome de sjögren (ss) 250, 251

Síndrome metabólica 115, 116, 117, 118, 119, 120

Sistema imunológico 58, 115, 117, 120

Sistema nacional de agravos de notificação (sinan) 140, 143

Situação de vulnerabilidade 90, 94, 95, 103

Superfície ocular 250, 254, 258

T

Tecnologia 66, 67, 69, 70, 137, 211, 231, 244

Tecnologias de informação e comunicação 208, 230

Teleatendimento 202, 204

Telemedicina 133, 170, 230, 232

Telessaúde 230, 236

Tempos pandêmicos 271

Terapêutica multidisciplinar 190

Teste de schirmer 250, 255

Testes oftalmológicos 250

Transmissão de informações 271, 279

Transtorno de ansiedade generalizada 190, 192

Tuberculose (tb) 151,152

$\mathbf{U}$

Unidade de saúde da família (usf) 52, 55

Uso das máscaras 43, 45, 48 
Vacina 28, 31, 32, 33, 34, 37, 39, 40, 70, 72, 159, 161, 183, 184, 186, 221

Variantes $28,30,31,34,35,38,49,78$

Vídeos educativos 215, 226

Violência contra a mulher $163,169,170,173,174$

$\mathbf{X}$

Xeroftalmia 250,251 


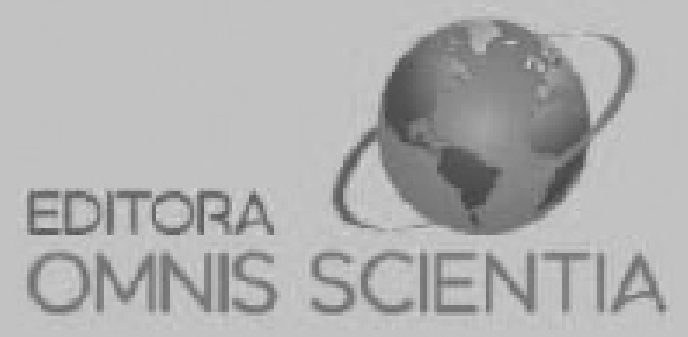

editoraomnisscientia@gmail.com M https://editoraomnisscientia.com.br/ @editora_omnis_scientia (0) https://wwww.facebook.com/omnis.scientia.9 $\boldsymbol{\oplus}$ +55 (87) 9656-3565 (C) 


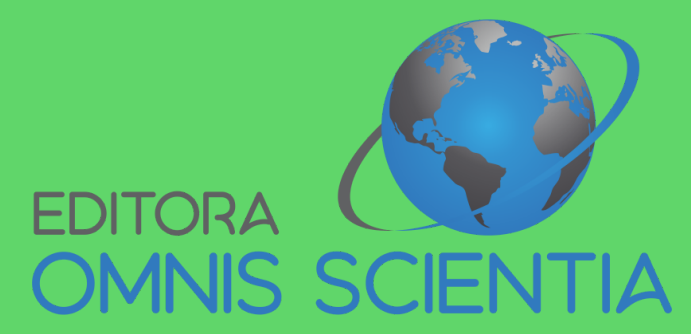

editoraomnisscientia@gmail.com M https://editoraomnisscientia.com.br/ (-) @editora_omnis_scientia (0) https://www.facebook.com/omnis.scientia.9 $\subsetneq$ +55 (87) 9656-3565 @ 